\title{
Ligamento suspensório da articulação metacarpo/metatarso falangianas nos eqüinos: aspectos evolutivos, anatômicos, histofisiológicos e das afecções
}

\author{
Suspensory ligament of equine metacarpus/metatarsus falangeal joint: evolutive anatomical, \\ histophysiological aspects and pathologies
}

\author{
Silke Verena Schwarzbach ${ }^{\mathrm{I}}$ Geane Maciel Pagliosa ${ }^{\mathrm{I}}$ Marcela Peixoto Roscoe $^{\mathrm{I}}$ \\ Geraldo Eleno Silveira Alves"
}

\section{- REVISÃo BIBLIOGRÁFICA -}

RESUMO

Esta revisão reúne e discute aspectos anatômicos, histofisiológicos e afecções do ligamento suspensório (LS) da articulação metacarpo/metatarso falangiana dos eqüinos. Considerou-se a importância desses aspectos para subsidiar o diagnóstico e o tratamento de afecções no LS, visto que freqüentemente animais portadores dessas doenças permanecem em atividades sob efeito de tratamentos paliativos que, apesar de proporcionarem alívio álgico, quase sempre não garantem a cura definitiva e, por conseguinte, não garantem o bem-estar absoluto do animal.

Palavras-chave: eqüino, ligamento suspensório, desmite, sistema locomotor.

\section{ABSTRACT}

This review discusses anatomical, histophysiological and pathological aspects of the suspensory ligament (SL) of metacarpal/metatarsal joint in the horse. This knowledge is important to aid in diagnosing and treating the SL diseases in the athlete horses, considering that affected animals remain on training activities under palliative treatments that insure pain relieve, but do not guarantee fully and definitively welfare of the horses.

Key words: equine, suspensory ligament, desmitis, locomotory system.

\section{INTRODUÇÃO}

O ligamento suspensório (LS) equivale ao músculo interósseo médio nos animais polidáctilos e é um importante componente do aparelho suspensório das articulações metacarpo ou metatarso falangiana (SISSON \& GROSSMAN, 1986; WILSON et al., 1991).

O aparelho suspensório (AS) é constituído pelos ossos sesamóides proximais, ligamento suspensório, sesamóides distais reto, oblíquos e cruzados (DYSON et al., 1995; GIBSON \& STEEL, 2002), intersesamóide, frenadores supra e infracarpal, tendões flexores superficial e profundo (WILSON et al., 1991; NOMINA ANATOMICA VETERINÁRIA, 1994). O conjunto de componentes do AS funciona de modo integrado, em que cada componente deve estar sadio e funcionar em equilíbrio biomecânico, a fim de não comprometer a integridade do conjunto. Qualquer lesão em um dos componentes gera risco de iniciar um ciclo etiopatogênico que atinge outros integrantes.

Identificar as estruturas afetadas, a natureza e a extensão das lesões requer conhecimentos específicos de anatomia, fisiologia e das doenças, os quais são os fundamentos para se efetuar o diagnóstico, o tratamento e o prognóstico (GIBSON \& STEEL, 2002), mesmo com a disponibilidade de recursos tecnológicos para exames complementares.

'Curso de Pós-graduação em Medicina Veterinária, área de concentração em Cirurgia de Grandes Animais, Escola Veterinária da Universidade Federal de Minas Gerais (EV/UFMG), Belo Horizonte, MG, Brasil.

IIDepartamento de Clínica e Cirurgia Veterinária, EV/UfMG. Av. Pres. Antônio Carlos, 6627, CP 567, 31270-901, Belo Horizonte, MG, Brasil. Email: geufmg@gmail.com. Autor para correspondência. 
As lesões do LS são consideradas como causas mais freqüentes de claudicação em eqüinos atletas (COWLES, 2000). Elas representam prejuízo econômico expressivo, sendo que o tratamento é demorado e as recidivas são freqüentes.

Esta revisão visa a abordar os aspectos evolutivos, anatômicos, histofisiológicos e as afecções do ligamento suspensório dos eqüinos, considerando a importância destes na prática da medicina esportiva eqüina, uma vez que, freqüentemente, lesões no LS não são diagnosticadas. Com isso, animais afetados permanecem em atividade sob efeito de tratamentos paliativos, o que favorece a cronicidade, amplificação das lesões e dependência de analgésicos para competir.

\section{Evolução}

A adaptação da espécie Equus caballus às transformações do ambiente representou um importante passo na evolução da espécie, quando a sustentação do corpo em cada membro de cinco dedos passou a ser exercida somente pelo terceiro (EDWARDS, 1994). Com isso, tecidos como o músculo interósseo também sofreram modificações intensas, passando a ser denominado ligamento suspensório, ligamento interósseo ou ligamento sesamoideano superior (WILSON et al., 1991). Acredita-se que ao longo da evolução o músculo interósseo médio dos eqüinos perdeu tecido muscular e adquiriu características de ligamento, recebendo a denominação de ligamento interósseo (SISSON \& GROSSMAN,1986; WILSON et al., 1991).

\section{Anatomia}

Visto transversalmente, o LS é circundado pelas faces palmar plantar dos ossos metacarpiano (Mtc) ou metatarsiano (Mtt) terceiros, as faces axiais dos ossos Mtc/Mtt II e IV, e a superfície dorsal do ligamento frenador (DENOIX, 1994). Nos membros torácicos, a origem do LS é na face palmar proximal do osso Mtc III (DENOIX, 1994) e na borda distal da cápsula articular do carpo. A origem do LS apresentase com duas cabeças, medial e lateral, separadas discretamente (BRAMLAGE et al., 1980), e confundese com as fibras do recesso palmar da cápsula articular, que se estende 1-2cm distalmente (DENOIX, 1994). Nos membros pélvicos, a origem do LS é na face plantar proximal do Mtt III e em alguns indivíduos nos ossos distais do tarso (GIBSON \& STEEL, 2002).

Nos membros torácicos e pélvicos o LS estende-se distalmente entre os ossos Mtc e Mtt II e IV, ou seja, no sulco metacárpico/metatársico (SISSON \& GROSSMAN, 1986), dividindo-se em dois ramos no terço distal do osso Mtc III e Mtt III.
A inserção dos ramos do LS ocorre nas superfícies abaxiais dos ossos sesamóides proximais correspondentes e prosseguem oblíqua e dorsalmente sobre a falange proximal, onde se unem ao tendão do músculo extensor digital comum, proximal à articulação interfalangeana proximal (WILSON et al., 1991). A irrigação do LS proximal provém do arco palmar profundo. O corpo do LS recebe um ramo da artéria nutrícia do osso Mtc II e das artérias metacarpianas palmares II e IV. Os ramos do LS são irrigados por derivações das artérias digital palmar comum II, metacarpianas palmares digital lateral e medial (NATALIE et al., 1999).

Na literatura consultada, os autores não foram consensuais sobre a inervação do ligamento suspensório (MUYLLE et al., 1998). DYSON (1995) afirmou que nos membros torácicos a inervação é derivada dos nervos metacarpianos lateral e medial, originados do ramo profundo do nervo ulnar. Um estudo posterior demonstrou que o nervo digital palmar lateral e os metacarpianos palmares contém fibras dos nervos mediano e ulnar (CASTRO et al., 2005). Nos membros pélvicos, o LS recebe inervação do nervo plantar lateral (GIBSON \& STEEL, 2002), que deriva do nervo tibial (DYSON, 200).

\section{Histologia}

O LS dos eqüinos é constituído de tecido conjuntivo e fibras musculares residuais, na taxa de 2 a 11\% (DYSON, 2000), o que pode explicar a variabilidade ecogênica das imagens do LS entre indivíduos durante exames de ultra-som. A presença de tecido conjuntivo frouxo situado ao longo do LS acomoda vasos e nervos (DYSON et al., 1995). O LS é composto por um sistema bilateral de fibras longas de colágeno, entrelaçadas em espiral e presas entre si por fibras cruzadas, que distalmente separam-se nos ramos do LS. As fibras musculares vestigiais estão inseridas entre as fibras longas de forma semicircular. Esse arranjo estrutural proporciona amortecimento de forças resultantes de vibrações durante a dinâmica da locomoção, evitando efeitos nocivos (ROONEY, 1973). A distribuição de fibras musculares vestigiais no LS é simétrica entre membros torácicos e pélvicos de um mesmo indivíduo, mas variável entre eqüinos da mesma raça (GIBSON \& STEEL, 2002). Consta na literatura que a proporção de fibras musculares não varia com a idade, mas existem opiniões divergentes quanto às mudanças de proporções em resposta à seqüência de treinamento (DENOIX, 1994; DYSON et al., 1995; REEF, 1998).

Estudos recentes mostraram que a matriz extracelular nos tendões do músculo flexor digital superficial, profundo e ligamento suspensório é 
composta de 60 a $70 \%$ de água, enquanto $80 \%$ da matéria seca contêm colágeno e outras proteínas, como matriz protéica oligomérica da cartilagem (MPOC) (SMITH, 2005).

Fisiologia

A função do AS é promover suporte e evitar a extensão excessiva das articulações metacarpo/ metatarso falangianas, sendo o LS um dos principais componentes do AS (WILSON et al., 1991).

A organização e a composição das fibras tendíneas e matriz extracelular garantem propriedade elástica para o funcionamento do AS. Assim, os eqüinos são capazes de realizar esforços sob velocidade e espaço de tempo, operando próximo ao limite de tolerância e, com isso, aumentando o risco de injúria (SMITH, 2005). O ligamento suspensório e os tendões flexores também são responsáveis na dissipação de forças quando ocorre sobrecarga no membro (DENOIX, 1994).

O exercício físico e a idade do animal podem exercer influência na matriz celular. A atividade metabólica dos tenócitos decresce em eqüinos acima de dois anos submetidos ao exercício, representando uma queda na capacidade de adaptação dos tendões e ligamentos após a maturidade óssea (SMITH, 2005).

Afecções e sinais clínicos

As injúrias ao LS foram inicialmente documentadas em animais de corrida das raças Standardbred e Thoroughbred (WILSON et al., 1991; PATTERSON-KANE et al., 1998). Com o advento do exame de ultra-som a partir da década de 80 , o diagnóstico de lesões no LS se tornou mais freqüente em eqüinos atletas de outras modalidades, tais como salto, adestramento (DYSON et al., 1995), enduro, pólo (MARKS et al., 1981), concurso completo de equitação (GIBSON \& STEEL, 2002) e trabalho de campo (JACKMANN, 2001).

A lesão por avulsão de fibras na origem do LS envolve a cabeça lateral ou medial do LS, na face palmar/plantar dos ossos Mtc/Mtt, podendo ser concomitante com uma fratura por tração nesses ossos (BRAMLAGE et al., 1980). Essas fraturas são comuns nos membros torácicos de eqüinos atletas e determinam claudicação aguda e severa ou esses animais possuem histórico de claudicação crônica recorrente sem diagnóstico definido (GIBSON \& STEEL, 2002). Nos animais portadores de avulsão, a dor é verificada pela palpação da área proximal palmar do metacarpo, podendo ser discreta em alguns casos. Nota-se aumento da claudicação ao trote ou marcha em círculos, quando o membro está do lado externo. Freqüentemente os testes de flexão do carpo/tarso acentuam a claudicação (DYSON et al., 1995; EDWARDS et al., 1995; JACKMANN, 2001).

A desmite do LS é caracterizada clinicamente por calor local, aumento de volume e dor na palpação, dependendo do estágio evolutivo da lesão. Eqüinos com afecções no AS freqüentemente apresentam alterações no balanceamento do casco, ou claudicação concomitante em outro membro (DYSON et al., 1995). A claudicação pode variar de discreta a severa examinando-se o animal ao passo. Nos casos discretos, a claudicação torna-se evidente ao trote ou à marcha em piso macio (MARKS et al., 1981).

A desmite proximal é uma afecção do LS que acomete o segmento proximal e pode não determinar sinais clínicos locais óbvios (GIBSON \& STEEL, 2002). A causa se resume na ruptura de fibras, resultantes de movimentos bruscos, por fuga repentina ou sobrecarga durante o exercício (UELTSCHI, 1989). Animais com desmite proximal freqüentemente apresentam claudicação no início do exercício ou durante a recepção do salto (MARKS et al., 1981).

A desmite do corpo ou ramos do LS é freqüente em eqüinos atletas e é a causa mais comum de descarte em eqüinos de salto (MARKS, 1999). Nos casos de desmite do corpo do LS, a claudicação pode ser discreta em casos agudos e apresentar dor moderada pela palpação quando o membro está elevado (GIBSON \& STEEL, 2002). Ocorre aumento de volume e temperatura na face palmar/plantar dos ossos Mtc/Mtt III (DYSON et al., 1995). A área do membro torácico comumente afetada é de 6-18 cm abaixo do osso acessório do carpo e no membro pélvico 15-24 cm distal da tuberosidade calcânea (COWLES, 2000).

A desmite dos ramos do LS é identificada pelo aumento de volume de um ou em ambos os ramos do LS. Pode estar acompanhada de edema periligamentar ou fibrose. A dor à palpação pode estar presente em casos agudos, mas a claudicação é variável e algumas vezes ausente (GIBSON \& STEEL, 2002).

Eqüinos acometidos por doença degenerativa do LS apresentam uma resposta de reparo cicatricial anormal. Enquanto o ligamento sofre degeneração, feixes de colágeno aderem-se para formar feixes maiores. Os fibroblastos ao centro destes feixes tornam-se isolados do aporte de sangue e degeneramse ou iniciam a produção de tecido cartilaginoso no lugar de colágeno. O ligamento perde elasticidade, tornando-se incapaz de oferecer suporte biomecânico pleno (MERO \& POOL, 2002). A degeneração é a afecção do LS que mais acomete eqüinos da raça Passo Fino, além de animais idosos de outras raças. 
Clinicamente, a degeneração caracteriza-se por uma hiperextensão progressiva da articulação metacarpo/metatarso falangeana, sendo mais incidente nos membros posteriores, decorrente de alongamento dos ligamentos suspensórios. Em casos de degeneração avançada do LS, exames radiográficos confirmam a posição anormal dos ossos sesamóides proximais em relação aos ossos Mtc e Mtt (MERO \& POOL, 2002).

A cicatrização das lesões no LS tem início após a hemorragia, a inflamação com remoção do tecido lesado por fagócitos, a proliferação e a migração de fibroblastos com produção de colágeno e posterior remodelação (GIBSON \& STEEL, 2002). As metaloproteinases aumentam suas concentrações durante a inflamação, lisam as fibras de colágeno tipo I originais (MCLLWRAIGHT, 1996), dando lugar a colágeno tipo III, em arranjos desorganizados de fibras. O tecido resultante não é equivalente ao original em resistência e elasticidade, sendo predisposto a lesões recidivantes. Em casos crônicos, a hemorragia é menos aparente e o edema se organiza em tecido fibroso, resultando em aumento de volume permanente do LS (GIBSON \& STEEL, 2002). As aderências entre o LS e os ossos Mtc III ou Mtt III podem causar dor e conseqüente claudicação (SCHNEIDER et al., 2005).

Apesar da cicatrização do LS ser considerada semelhante ao que ocorre no tendão flexor superficial, estudos mostraram que a qualidade de reparo do LS é inferior (REEF, 1998). Além disso, a evolução da cicatrização no LS é relativamente demorada, com alterações persistentes identificáveis à ultra-sonografia até seis meses após o início do tratamento (RAPP et al., 1992).

No diagnóstico das afecções no LS, devem ser considerados os sinais clínicos pela inspeção e palpação local, além de técnicas auxiliares, tais como bloqueio anestésico regional, ultra-sonografia, radiografia, cintilografia (DYSON et al., 1995; GIBSON \& STEEL, 2002) e ressonância magnética (SCHNEIDER et al., 2005). Para realizar a palpação do IS, o carpo/ tarso deve estar flexionado. A borda lateral proximal do LS de eqüinos é mais sensível à palpação que a medial (MARKS et al., 1981).

Descreveu-se que o bloqueio anestésico do nervo palmar lateral, distalmente ao osso acessório do carpo, é importante para localizar a origem da claudicação (MARKS et al., 1981; FORD et al., 1989; MUYLLE et al., 1998; GIBSON \& STEEL, 2002). O bloqueio anestésico alto do nervo palmar em quatro pontos é realizado nos sulcos entre o tendão flexor digital profundo e LS, atingindo os nervos digitais palmares e metacarpianos (KEG et al., 1996; CASTRO et al., 2005). Outros autores, entre estes KEG et al.
(1996), afirmaram que o bloqueio anestésico realizado $10 \mathrm{~cm}$ proximal ao osso acessório do carpo, no sulco entre os músculos flexor carpo-ulnar e ulnar lateral, resulta em desensibilização de todo o LS. CASTRO et al. (2005) descreveram uma técnica de bloqueio anestésico, realizada medialmente ao osso acessório do carpo, utilizando um volume de anestésico significativamente menor.

Ao exame radiográfico, fraturas proximais do osso Mtc III na origem do LS são identificadas mais facilmente nas projeções oblíquas e lateromedial com o carpo flexionado. Fraturas não-deslocadas são vistas com aspecto de "V" ou linhas lucentes semicirculares na projeção antero-posterior, enquanto fraturas deslocadas são identificadas nas projeções lateromedial (DYSON et al., 1995).

Nos casos crônicos de desmite proximal, áreas de esclerose na região proximal dos ossos Mtc III/Mtt III são identificadas por exames radiográficos que evidenciam alteração do padrão trabecular ósseo (UELTSCHI, 1989), antesófitos na projeção lateromedial (DYSON, 1994) e periostite ossificante na projeção antero-posterior (DYSON et al., 1995).

O exame de ultra-som deve ser realizado com um transdutor linear de $7,5 \mathrm{MHz}$ e conector, pois minimiza artefatos ao possibilitar maior área de contato com as estruturas (DYSON et al., 1995). O membro afetado sempre deve ser comparado com o contralateral. A imagem do LS sadio apresenta ecogenicidade mista, devido ao tecido adiposo e às fibras musculares vestigiais entremeadas no tecido conjuntivo denso do ligamento (STEYN \& McLLWRAIGHT, 1991). Nos membros torácicos, o LS apresenta forma retangular e nos posteriores sua forma é arredondada na imagem transversal, partindo de duas cabeças separadas por uma faixa menos ecogênica (DYSON et al., 1995).

A cintilografia detecta precocemente fissuras ósseas, que não são identificadas ao exame radiográfico (EDWARDS et al., 1995). Com o advento da ressonância magnética foi possível identificar uma estreita relação entre exostose ou reações periosteais discretas nos ossos Mtc/Mtt III e desmite do LS.

O tratamento da desmite do LS ainda é um desafio, pois estudos clínicos avaliando as respostas terapêuticas não apresentam progressos satisfatórios (DYSON, 1991). BRAMLAGE et al. (1980) recomendaram repouso limitado para eqüinos com avulsão da inserção do LS, enquanto a maioria dos autores consultados recomendou repouso de 3 a 9 meses com exercício controlado (DYSON et al., 1995). O exercício ao passo deve ser realizado durante as 12 primeiras semanas com aumento da intensidade de acordo com a evolução da claudicação e imagens de ultra-som seriadas. 
Alguns autores relataram a administração de corticosteróides, ácido hialurônico ou células tronco adultas intralesional, revulsivos e, mais recentemente, ondas de choque (COWLES, 2000; MCCLURE \& EVANS, 2002) e neurectomia do nervo tibial e ramo profundo do nervo palmar lateral (CROWE et al., 2002). A terapia por ondas de choque tem sido usada para controle da dor na desmite proximal do LS, em que pulsos de pressão acústica aplicados sobre o local afetado geram forças que agem nas interfaces celulares. A administração de antiinflamatórios pode ser efetuada concomitantemente às terapias locais (GIBSON \& STEEL, 2002).

Estudos recentes, entre estes de DAHLGREN (2005), consideram três fatores básicos da regeneração tissular importantes para um tratamento eficaz: a introdução de uma estrutura de sustentação tridimensional para orientar a cicatrização tecidual; células precursoras capazes de sofrerem diferenciação em células de tendão ou de colágeno e fatores de crescimento e citocinas para orientação da matriz celular.

Nos casos de desmite proximal do LS nos membros pélvicos, o prognóstico é menos favorável que nos torácicos, mas pode estar relacionado com o intervalo entre o início da claudicação e o diagnóstico (DYSON et al., 1995). CROWE et al. (2002) consideraram o prognóstico da desmite proximal do membro pélvico reservado. Segundo DYSON et al. (1995), 86\% dos eqüinos com desmite proximal do LS dos membros torácicos, avaliados em um estudo, retornaram ao treinamento intensivo após um programa de repouso e exercício controlado, enquanto que apenas $17 \%$ dos eqüinos com desmite proximal do LS no membro pélvico voltou ao desempenho anterior.

O prognóstico da desmite no corpo do LS depende do tipo de serviço exercido pelo eqüino e da severidade da injúria. Apesar do retorno dos animais acometidos às pistas de corrida, a recorrência é alta (COULBOURNE \& YOVICH, 1994). O prognóstico é favorável em animais com lesões discretas, mas é reservado em animais com desmite do ramo do LS em membros pélvicos (GIBSON \& STEEL, 2002).

\section{CONCLUSÕES}

As afecções do ligamento suspensório constituem um obstáculo freqüente para vida atlética do eqüino. A recuperação é influenciada por diversos fatores, entre eles a necessidade de diagnóstico e tratamento oportuno e adequado, já que o repouso absoluto é praticamente impossível na espécie. As afecções do LS acarretam prejuízos econômicos e de bem-estar importantes, além de não permitirem que eqüinos atletas atinjam seu limite de desempenho. Além da baixa freqüência de diagnóstico e da interpretação difícil dos sinais clínicos, as afecções no LS sempre exigem exames complementares.

O LS adaptou-se anatômica e fisiologicamente durante a evolução da espécie, tornando-se uma estrutura especializada para suportar esforços extremos de longa duração, próximos aos limites de tolerância para a ocorrência de lesões. A organização anatômica do LS torna as células do ligamento sensíveis às alterações fisiológicas mínimas do organismo. O mecanismo de reparo tecidual de tendões e ligamentos em resposta à injúria cito e histológica ainda não está completamente elucidado, o que demanda mais pesquisas na área.

Infelizmente as opções e respostas terapêuticas existentes para injúrias do LS ainda não apresentam progressos satisfatórios, motivo que desperta interesse para o desenvolvimento de pesquisas para tratamento das afecções ligamentares.

\section{REFERÊNCIAS}

BRAMLAGE, L.R. et al. Avulsion fractures of the origin of the suspensory ligament in the horse. Journal American Veterinary Medical Association, v.176, p.1004-1010, 1980.

CASTRO, F.A. et al. A new approach for perineural injection of the lateral palmar nerve in the horse. Veterinary Surgery, v.34, p.539, 2005

COULBOURNE, C.M; YOVICH, J.V. Suspensory ligament injuries in racing horses: ultrasonographic diagnosis and long term follow up. Australian Equine Veterinary Journal, v.12, p.119-128, 1994.

COWLES, R.R. Proximal suspensory desmitis- a qualitative survey. Proceedings American Association Equine Practice, v.46, p.143-144, 2000.

CROWE, O. et al. Treatment of chronic hindlimb proximal suspensory desmitis by radial extracorporeal shockwave therapy. Proceedings American Association Equine Practice, v.48, p.322-325, 2002.

DAHLGREN, L.A. A review of treatment options for equine tendon and ligament injuries: what's new and how do they work? In: ANNUAL CONVENTION OF THE AMERICAN ASSOCIATION OF EQUINE PRACTIONERS, 2005, Seattle. American Association Equine Practice, Lexington, International Veterinary Information Service. Capturado em 22 mar. 2007. Online. Disponível na Internet http://www. ivis.org.

DENOIX, J.M. Functional anatomy of tendons and ligaments in the distal limb (manus and pes). Veterinary Clinics of North América: Equine Practice, v.10, p.213-321, 1994. 
DYSON, S. Proximal suspensory desmitis; clinical, ultrasonographic and radiographic features. Equine Veterinary Journal, v.23, p.25-31, 1991.

DYSON, S. Proximal suspensory desmitis in the hindlimb: 42 cases. British Veterinary Journal, v.150, p.279-291, 1994.

DYSON, S. Proximal suspensory desmitis in the hindlimb. Equine Veterinary Education, v.7, p.275-278, 1995.

DYSON, S. Proximal suspensory desmitis in the forelimb and the hindlimb. Proceedings American Association Equine Practice, v.46, p.137-142, 2000.

DYSON, S. et al. Suspensory ligament desmitis. Veterinary Clinics of North América: Equine Practice, v.11, p.177215, 1995.

EDWARDS, E.H. O grande livro do cavalo. Hong Kong: Contralivros, 1994. 240p.

EDWARDS, R.B. et al. Scintigraphy for diagnosis of avulsions of the origin of the suspensory ligament in horses: 51 cases (1980- 1993). Journal American Veterinary Medical Association, v.207, p.608-611, 1995.

FORD, T.S. et al. A comparison of methods for proximal palmar analgesia in horses. Veterinary Surgery, v.18, p.146150, 1989.

GIBSON, K.T.; STEEL, C.M. Conditions of the suspensory ligament causing lameness in horses. Equine Veterinary Education, v.14, p.39-50, 2002.

JACKMANN, B.R. Common lameness in the cutting and reining horse. Proceedings American Association Equine Practice, v.47, p.6-11, 2001.

KEG, P.R. et al. The effect of the high palmar nerve block and the ulnar nerve block on lameness provoked by a collagenaseinduced tendonitis of the lateral branch of the suspensory ligament. Veterinary Quarterly., v.18, p.103-105, 1996.

MACCLURE, S.; EVANS, R.B. In vivo evaluation of extracorporeal shock wave therapy for collagenase induced suspensory ligament desmitis. Proceedings American Association Equine Practice, v.48, p.378-380, 2002.

McLLWRAIGHT, C.W. Joint disease in the horse. Philadelphia: Saunders, 1996. 490p.

MARKS, D. Prepurchase examination of jumpers and dressage horses. Proceedings American Association Equine Practice, v.45, p.4-12, 1999.

MARKS, D. et al. Lameness resulting from high suspensory disease (HSD) in the horse. Proceedings American Association Equine Practice, v.24, p.493-497, 1981.

MERO, J.L.; POOL, R.R. Twenty cases of degenerative suspensory ligamenet desmitis in Peruvian Paso horses. Proceedings American Association Equine Practice, v.48, p.329-334, 2002.
MUYLLE, S. et al. Histological study of the innervation of the suspensory ligament of the forelimb of the horse. Veterinary Record, v.142, p.606-610, 1998.

NATALIE, M.V. et al. Irrigación del músculo interosseo y del ligamento accessorio del músculo flexor digital profundo en la mano del caballo. Archivo Medico Veterinario, v.31, p.2012006, 1999.

NOMINA ANATOMICA VETERINARIA. 4. ed. Ithaca: Word Association on Veterinary Anatomists, 1994. Capturado em 12 mar. 2006. Online. Disponível na Internet. http://www.wavaamav.org

PATTERSON-KANE, J.C. et al. Effects on training collagen fibril populations in the suspensory ligament and deep digital flexor tendon of young Thoroughbreds. American Journal Veterinary Research, v.59, p.64-68, 1998.

RAPP H.J. et al. Überlegungen zur diagnose von tendinitiden beim pferd sowie deren behandlunsprinzipien. Praktischer Tierarzt, v.1, p.13-20, 1992.

REEF, V. Musculoskeletal ultrasonografy. In: REEF, V. Equine diagnostic ultrasound. Philadelphia: Saunders, 1998. p.39186.

ROONEY, J. Functional anatomy of the equine suspensory ligament. Modern Veterinary Practice, v.54, p.43-45, 1973.

SCHNEIDER, R.K. et al. Magnetic resonance imaging evaluation of horses with lameness problems. In: ANNUAL CONVENTION OF THE AMERICAN ASSOCIATION OF EQUINE PRACTIONERS, 51., 2005, Seattle. Proceedings... Lexington: International Veterinary Information Service. Capturado em 26 mar. 2006. Online. Disponível na Internet. http://www. ivis.org.

SISSON, S.; GROSSMAN, J.D. Anatomia dos animais domésticos. 5.ed. Rio de Janeiro: Guanabara Koogan, 1986. p.42-44.

SMITH, R.K. Physiology of tendon and ligament. In: CONGRESS ON EQUINE MEDICINE \& SURGERY IN GENEVA, 9., 2005, Geneva. Proceedings... Lexington: International Veterinary Information Service. Capturado em 12 dez. 2006. Online. Disponível na Internet http://www. ivis.org.

STEYN, P.F.; MCLLWRAIGHT, C.W. The ultrassonographic examination of the palmar metacarpal tendons and ligaments of the equine digit: a review. Equine Practice, v.13, p.24-31, 1991.

UELTSCHI, G. Zur diagnose von interosseusläsionen an der ursprungsstelle. Pferdeheilkunde, v.5, p.65-69, 1989.

WILSON, D.A. et al. Composition and morphologic features of the interosseous muscle in Standardbreds and Thoroughbreds. American Journal of Veterinary Research, v.52, p.13331339, 1991. 